\title{
Scattering and Bound State Solutions of the Yukawa Potential within the Dirac Equation
}

\author{
F. Pakdel, ${ }^{1}$ A. A. Rajabi, ${ }^{1}$ and M. Hamzavi ${ }^{2}$ \\ ${ }^{1}$ Deparment of Physics, University of Shahrood, Shahrood, Iran \\ ${ }^{2}$ Deparment of Physics, University of Zanjan, Zanjan, Iran \\ Correspondence should be addressed to M. Hamzavi; majid.hamzavi@gmail.com
}

Received 16 November 2013; Accepted 23 January 2014; Published 17 March 2014

Academic Editor: Shi-Hai Dong

Copyright (c) 2014 F. Pakdel et al. This is an open access article distributed under the Creative Commons Attribution License, which permits unrestricted use, distribution, and reproduction in any medium, provided the original work is properly cited. The publication of this article was funded by $\mathrm{SCOAP}^{3}$.

In the presence of spin symmetry case, we obtain bound and scattering states solutions of the Dirac equation for the equal scalar and vector Yukawa potentials for any spin-orbit quantum number $\kappa$. The approximate analytical solutions are presented for the bound and scattering states and scattering phase shifts.

\section{Introduction}

For studying the quantum mechanical systems, it is necessary to pay attention to two points. These two points are to study bound states to take the necessary information about the system under consideration and also solving scattering states for a system under the effect of a potential. Solving both of these problems gives us complete information about a quantum mechanical system under consideration.

The solutions of scattering and/or bound state problem have been investigated for the well-known potentials by applying different methods [1-10]. The analytical scattering state solution of the $l$-wave Schrödinger Equation for the Eckart potential has been obtained in [11]. The solution of the Schrödinger equation for the modified Morse potential has been studied by Wei and Chen [12]. Rojas and Villalba have found the solutions of the Klein-Gordon equation for one-dimensional Wood-Saxon potential by hypergeometric functions [13]. The exact solutions of scattering state have been studied for the $s$-wave Schrödinger equation with the Manning-Rosen potential by using standard method [14]. Low momentum scattering states of the Dirac equation have been studied in [15]. Properties of scattering state solutions of the Klein-Gordon equation Coulomb scalar plus vector potential have been studied in [16].
In this work, we have studied bound state and scattering state of the Dirac equation with the Yukawa potential. The Dirac equation describes the particle dynamics in the relativistic quantum mechanics $[17,18]$. Thus, solving the Dirac equation is very significant in describing the nuclear shell structure $[19,20]$. Also the Yukawa potential has many applications in different areas of physics like high-energy physics [21] and atomic, molecular, and plasma physics [22].

This paper is organized as follows. In Section 2, we briefly introduce the Dirac equation with scalar and vector potential with any spin-orbit quantum number $\kappa$. The bound and scattering states of the Yukawa potential within the Dirac equation are presented in Section 3. Finally, our concluding remarks are given in Section 4.

\section{Dirac Equation with Scalar and Vector Potential}

The Dirac equation with scalar and vector potential $(S(r)$ and $V(r))$ is $[\hbar=c=1]$

$$
[\alpha \cdot p+\beta(M+S(r))] \psi(r)=(E-V(r)) \psi(r),
$$


where $E$ is the relativistic energy of the system and $P=-i \nabla$ is the three-dimensional momentum operator. $\alpha$ and $\beta$ are the usual $4 \times 4$ Dirac matrices given as

$$
\alpha=\left(\begin{array}{ll}
0 & 1 \\
1 & 0
\end{array}\right), \quad \beta=\left(\begin{array}{cc}
I & 0 \\
0 & -I
\end{array}\right), \quad i=1,2,3,
$$

where $I$ is the $2 \times 2$ unitary matrix and the three $2 \times 2$ Pauli matrices $\sigma_{i}$ are given as

$$
\sigma_{1}=\left(\begin{array}{ll}
0 & 1 \\
1 & 0
\end{array}\right), \quad \sigma_{2}=\left(\begin{array}{cc}
0 & -i \\
-i & 0
\end{array}\right), \quad \sigma_{3}=\left(\begin{array}{cc}
1 & 0 \\
0 & -1
\end{array}\right)
$$

where $L$ is the orbital angular momentum of the spherical nucleons and the total angular momentum operator $J$ and spin-orbit $K=(\sigma \cdot L+1)$ commute with Dirac Hamiltonian. The eigenvalues of spin-orbit coupling operator are $k=(j+$ $(1 / 2))>0$ and $k=-(j+(1 / 2))<0$ for unaligned spin $j=l-(1 / 2)$ and the aligned spin $j=l+(1 / 2)$, respectively. Thus, in the Pauli-Dirac representation,

$$
\psi_{n k}(r)=\left(\begin{array}{c}
f_{n k}(r) \\
g_{n k}(r)
\end{array}\right)=\left(\begin{array}{c}
\frac{F_{n k}(r)}{r} Y_{j m}^{l}(\theta, \varphi) \\
i \frac{G_{n k}(r)}{r} Y_{j m}^{\tau}(\theta, \varphi)
\end{array}\right),
$$

where $f_{n k}(r)$ is the upper component and $g_{n k}(r)$ is the lower component of the Dirac spinors. $Y_{j m}^{l}(\theta, \varphi)$ and $Y_{j m}^{\tau}(\theta, \varphi)$ are spin and pseudospin spherical harmonics and $m$ is the projection of the angular momentum on the $z$-axis. Substituting (4) into (1), one obtains two coupled differential equations for the upper and the lower radial wave functions as follows:

$$
\begin{aligned}
& \left(\frac{d}{d r}+\frac{k}{r}\right) F_{n k}(r)=\left[M+E_{n k}-\Delta(r)\right] G_{n k}(r), \\
& \left(\frac{d}{d r}-\frac{k}{r}\right) G_{n k}(r)=\left[M-E_{n k}+\Sigma(r)\right] F_{n k}(r),
\end{aligned}
$$

where

$$
\begin{aligned}
& \Delta(r)=V(r)-S(r), \\
& \Sigma(r)=V(r)+S(r) .
\end{aligned}
$$

Solving (5) leads to a second-order Schrödinger-like differential equation for the upper and the lower components of the Dirac wavefunctions as follows:

$$
\begin{aligned}
{\left[\frac{d^{2}}{d r^{2}}-\frac{k(k-1)}{r^{2}}\right] G_{n k}(r) } & \\
+ & {\left[-\left(M+E_{n k}-\Delta(r)\right)\left(M-E_{n k}+\Sigma(r)\right)\right.} \\
& \left.-\frac{(d \Sigma(r) / d r)(d / d r-k / r)}{M-E_{n k}+\Sigma(r)}\right] G_{n k}(r)=0,
\end{aligned}
$$

$$
\begin{aligned}
{\left[\frac{d^{2}}{d r^{2}}-\frac{k(k+1)}{r^{2}}\right] F_{n k}(r) } \\
+\left[-\left(M+E_{n k}-\Delta(r)\right)\left(M-E_{n k}+\Sigma(r)\right)\right. \\
\left.+\frac{(d \Delta(r) / d r)(d / d r+k / r)}{M+E_{n k}-\Delta(r)}\right] F_{n k}(r)=0,
\end{aligned}
$$

where $k(k-1)=\widetilde{l}(\widetilde{l}+1)$ and $k(k+1)=l(l+1)$.

When scalar potential $S(r)$ is equal to the vector potential $V(r),(7 \mathrm{~b})$ becomes

$$
\begin{gathered}
{\left[\frac{d^{2}}{d r^{2}}-\frac{k(k+1)}{r^{2}}-2\left(E_{n k}+M\right) V(r)\right] F_{n k}(r)} \\
=\left[M^{2}-E_{n k}^{2}\right] F_{n k}(r)
\end{gathered}
$$

and with (5), we have

$$
G_{n k}(r)=\frac{1}{M+E_{n k}}\left[\frac{d}{d r}+\frac{k}{r}\right] F_{n k}(r) .
$$

These are two equations with equal scalar and vector potential that we have used in this work.

\section{Dirac Equation with the Yukawa Potential}

According to (8), we have

$$
\begin{aligned}
& {\left[\frac{d^{2}}{d r^{2}}-\frac{k(k+1)}{r^{2}}-2\left(E_{n k}+M\right) V(r)\right.} \\
& \left.+E_{n k}^{2}-M^{2}\right] F_{n k}(r)=0 .
\end{aligned}
$$

The Yukawa potential is

$$
V(r)=-\frac{A}{r} e^{-\alpha r},
$$

where $\alpha$ is the screening parameter and $A$ is the strength of the potential $[21,23]$.

Instead of the centrifugal term in (10), the following approximation has been used in recent years:

$$
\frac{1}{r^{2}} \approx 4 \alpha^{2} \frac{e^{-2 \alpha r}}{\left(1-e^{-2 \alpha r}\right)^{2}}
$$

that is, it has a good accuracy for small values of the potential parameter [24, 25].

Substituting (11) and (12) into (10), we obtain the following form of the wave equation:

$$
\begin{aligned}
& {\left[\frac{d^{2}}{d r^{2}}-4 \alpha^{2} k(k+1) \frac{e^{-2 \alpha r}}{\left(1-e^{-2 \alpha r}\right)^{2}}+4 A \alpha\left(E_{n k}+M\right)\right.} \\
& \left.\times \frac{e^{-2 \alpha r}}{1-e^{-2 \alpha r}}+E_{n k}^{2}-M^{2}\right] F_{n k}(r)=0 .
\end{aligned}
$$


3.1. Bound State Solutions. For the bound state, by taking the following variable $(z \rightarrow 1$ for $r \rightarrow 0$ and $z \rightarrow 0$ for $r \rightarrow \infty$ )

$$
z=e^{-2 \alpha r}
$$

one can obtain (13) in the following form:

$$
\begin{gathered}
{\left[z(1-z) \frac{d^{2}}{d z^{2}}+(1-z) \frac{d}{d z}+\frac{1}{z(1-z)}\right.} \\
\times\left\{-k(k+1) z+\frac{A}{\alpha}\left(E_{n k}+M\right)\left(z-z^{2}\right)\right. \\
\left.\left.+\frac{E_{n k}^{2}-M^{2}}{4 \alpha^{2}}(1-z)^{2}\right\}\right] F_{n k}(z)=0 .
\end{gathered}
$$

Taking the form of the wave function

$$
F_{n k}(z)=z^{\mu}(1-z)^{\nu} f_{n k}(z)
$$

and substituting this equation into (15), one gets a hypergeometric-type equation as follows [26]:

$$
\begin{gathered}
z(1-z) f_{n k}^{\prime \prime}(z)+(2 \mu+1-(2 \mu+2 \nu+1) z) f_{n k}^{\prime}(z) \\
+\left[\mu(\mu-1) \frac{(1-z)}{z}-2 \mu \nu\right. \\
+\nu(\nu-1) \frac{z}{1-z}+\mu \frac{(1-z)}{z}-v \\
-k(k+1) \frac{1}{(1-z)}+\frac{A}{\alpha}\left(E_{n k}+M\right) \\
\left.+\frac{\left(E_{n k}^{2}-M^{2}\right)}{4 \alpha^{2}} \frac{(1-z)}{z}\right] f_{n k}(z)=0,
\end{gathered}
$$

where

$$
\mu=i \sqrt{\frac{\left(E_{n k}^{2}-M^{2}\right)}{4 \alpha^{2}}}, \quad v(\nu-1)=k(k+1) \quad(\nu>0) .
$$

Comparing (17) with the hypergeometric equation of the form [26]

$$
z(1-z) f_{n k}^{\prime \prime}(z)+[c-(a+b+1) z] f_{n k}^{\prime}(z)-a b f_{n k}(z)=0
$$

we can obtain the wavefunction as the hypergeometric function:

$$
f_{n k}(z)={ }_{2} F_{1}\left(a_{n k}, b_{n k}, c_{n k} ; z\right)
$$

where

$$
\begin{gathered}
a_{n k}=\mu+\nu+\sqrt{\frac{A}{\alpha}\left(E_{n k}+M\right)-\frac{\left(E_{n k}^{2}-M^{2}\right)}{4 \alpha^{2}}}, \\
b_{n k}=\mu+\nu-\sqrt{\frac{A}{\alpha}\left(E_{n k}+M\right)-\frac{\left(E_{n k}^{2}-M^{2}\right)}{4 \alpha^{2}}}, \\
c_{n k}=2 \mu+1 .
\end{gathered}
$$

Then, with (14) and (16), we have the upper-spinor component of wavefunction for the Dirac equation with the Yukawa potential as follows:

$$
\begin{aligned}
F_{n k}(r)= & C_{n k} e^{-2 \alpha \mu r}\left(1-e^{-2 \alpha r}\right)^{\nu} \\
& \times{ }_{2} F_{1}\left(a_{n k}, b_{n k}, c_{n k} ; e^{-2 \alpha r}\right),
\end{aligned}
$$

where $C_{n k}$ is the normalization constant. With (9), the lowerspinor component can be obtained as

$$
\begin{aligned}
G_{n k}(r)= & \frac{C_{n k}}{M+E_{n k}}\left[\frac{d}{d r}+\frac{k}{r}\right] e^{-2 \alpha \mu r}\left(1-e^{-2 \alpha r}\right)^{v} \\
& \times{ }_{2} F_{1}\left(a_{n k}, b_{n k}, c_{n k} ; e^{-2 \alpha r}\right) .
\end{aligned}
$$

Therefore,

$$
\begin{aligned}
G_{n k}(r) & \\
=\frac{C_{n k}}{M+E_{n k}}[ & k \frac{e^{-2 \alpha \mu r}}{r}\left(1-e^{-2 \alpha r}\right)^{\nu} \\
& \times{ }_{2} F_{1}\left(a_{n k}, b_{n k}, c_{n k} ; e^{-2 \alpha r}\right) \\
& -2 \alpha \mu e^{-2 \alpha \mu r}\left(1-e^{-2 \alpha r}\right)^{\nu} \\
& \times{ }_{2} F_{1}\left(a_{n k}, b_{n k}, c_{n k} ; e^{-2 \alpha r}\right) \\
& +2 \alpha \nu e^{-2 \alpha(\mu+1) r}\left(1-e^{-2 \alpha r}\right)^{\nu-1} \\
& \times{ }_{2} F_{1}\left(a_{n k}, b_{n k}, c_{n k} ; e^{-2 \alpha r}\right) \\
& +\left(\frac{a_{n k} b_{n k}}{c_{n k}}\right) e^{-2 \alpha \mu r}\left(1-e^{-2 \alpha r}\right)^{\nu} \\
& \left.\times{ }_{2} F_{1}\left(a_{n k}+1, b_{n k}+1, c_{n k}+1 ; e^{-2 \alpha r}\right)\right] .
\end{aligned}
$$

Finally, the spinor wave function under the condition of equal scalar and vector potentials with (4), (7b), (22), and (24) becomes

$$
\begin{aligned}
\Psi(r)= & \left(\begin{array}{c}
\frac{F_{n k}(r)}{r} Y_{j m}^{l}(\theta, \varphi) \\
i \frac{G_{n k}(r)}{r} Y_{j m}^{\tilde{l}}(\theta, \varphi)
\end{array}\right) \\
= & \frac{C_{n k}}{r}\left(\frac{-i}{M+E_{n k}}(\sigma \cdot \widehat{r}) \Re(r)\right) e^{-2 \alpha \mu r}\left(1-e^{-2 \alpha r}\right)^{\nu} \\
& \times Y_{j m}^{l}(\theta, \varphi)_{2} F_{1}\left(a_{n k}, b_{n k}, c_{n k} ; e^{-2 \alpha r}\right), \\
\Re(r)= & \frac{k}{r}-2 \alpha \mu+2 \alpha v e^{-2 \alpha r}\left(1-e^{-2 \alpha r}\right)^{-1} \\
& +\left(\frac{a_{n k} b_{n k}}{c_{n k}}\right) \frac{{ }_{2} F_{1}\left(a_{n k}+1, b_{n k}+1, c_{n k}+1 ; e^{-2 \alpha r}\right)}{{ }_{2} F_{1}\left(a_{n k}, b_{n k}, c_{n k} ; e^{-2 \alpha r}\right)} .
\end{aligned}
$$


By considering the finiteness of the solution, the quantum condition is given by

$$
\mu+\nu-\sqrt{\frac{A}{\alpha}\left(E_{n k}+M\right)-\frac{\left(E_{n k}^{2}-M^{2}\right)}{4 \alpha^{2}}}=-n, \quad n=0,1,2,3 .
$$

It is the energy eigenvalue equation of bound state for the upper component of the Dirac equation with the Yukawa potential.

3.2. Scattering State. Now, we turn to solve (10) for scattering state. For this purpose, we use a new variable as follows:

$$
\begin{aligned}
y & =1-e^{-2 \alpha r}, \quad y=1-z, \\
(r \longrightarrow \infty, \quad y \longrightarrow 1, \quad r \longrightarrow 0, & y \longrightarrow 0)
\end{aligned}
$$

and obtain

$$
\begin{aligned}
& {\left[y(1-y) \frac{d^{2}}{d y^{2}}-y \frac{d}{d y}-k(k+1) \frac{1}{y}\right.} \\
& \left.+\frac{A}{\alpha}\left(E_{n k}+M\right)+\frac{y}{4 \alpha^{2}(1-y)}\left(E_{n k}^{2}-M^{2}\right)\right] F_{n k}(y)=0 .
\end{aligned}
$$

Considering the boundary condition of the scattering state, we take the following trial wavefunction:

$$
F_{n k}(y)=y^{\eta}(1-y)^{\beta} f_{n k}(y) \text {. }
$$

And inserting this equation into (28), we obtain the following equation:

$$
\begin{aligned}
y(1-y) & f_{n k}^{\prime \prime}(y)+[2 \eta-(2 \eta+2 \beta+1) y] f_{n k}^{\prime}(y) \\
+ & {\left[\eta(\eta-1) \frac{(1-y)}{y}-2 \beta \eta+\beta(\beta-1) \frac{y}{(1-y)}-\eta\right.} \\
+ & \beta \frac{y}{(1-y)}-k(k+1) \frac{1}{y}+\frac{A}{\alpha}\left(E_{n k}+M\right) \\
& \left.+\frac{1}{4 \alpha^{2}}\left(E_{n k}^{2}-M^{2}\right) \frac{y}{(1-y)}\right] f_{n k}(y)=0,
\end{aligned}
$$

where

$$
\begin{aligned}
& \beta=i \sqrt{\frac{\left(E_{n k}^{2}-M^{2}\right)}{4 \alpha^{2}}}, \quad \eta(\eta-1)=k(k+1) \\
& (\eta>0) \text {. }
\end{aligned}
$$

This equation is a hypergeometric type. Thus, (29) is as follows: ( $N_{n k}$ is the normalization constant)

$$
F_{n k}(y)=N_{n k} y^{\eta}(1-y)_{2}^{\beta} F_{1}(a, b, c ; y)
$$

that is, the upper component of wavefunction. The lower component is

$$
\begin{aligned}
G_{n k}(r)= & \frac{N_{n k}}{M+E_{n k}}\left[\frac{d}{d r}+\frac{k}{r}\right] e^{-2 \alpha \beta r}\left(1-e^{-2 \alpha r}\right)^{\eta} \\
& \times{ }_{2} F_{1}\left(a_{n k}, b_{n k}, c_{n k} ; 1-e^{-2 \alpha r}\right) .
\end{aligned}
$$

And therefore, total wavefunction of scattering state with (4), (7b), (32), and (33) is

$$
\begin{aligned}
& \psi(r)=\left(\begin{array}{c}
\frac{F_{n k}(r)}{r} Y_{j m}^{l}(\theta, \varphi) \\
i \frac{G_{n k}(r)}{r} Y_{j m}^{\tilde{l}}(\theta, \varphi)
\end{array}\right) \\
& =\frac{N_{n k}}{r}\left(\begin{array}{c}
1 \\
\frac{-i}{E_{n k}+M}(\sigma \cdot \widehat{r}) \mathfrak{R}(r)
\end{array}\right) \\
& \times e^{-2 \alpha \beta r}\left(1-e^{-2 \alpha r}\right)^{\eta} Y_{j m}^{l}(\theta, \varphi) \\
& \times{ }_{2} F_{1}\left(a_{n k}, b_{n k}, c_{n k} ; 1-e^{-2 \alpha r}\right) \text {, }
\end{aligned}
$$

where

$$
\begin{aligned}
& \mathfrak{R}(r)= \frac{k}{r}-2 \alpha \beta+2 \alpha \eta e^{-2 \alpha r}\left(1-e^{-2 \alpha r}\right)^{-1}+\left(\frac{a_{n k} b_{n k}}{c_{n k}}\right) \\
& \times \frac{{ }_{2} F_{1}\left(a_{n k}+1, b_{n k}+1, c_{n k}+1 ; 1-e^{-2 \alpha r}\right)}{{ }_{2} F_{1}\left(a_{n k}, b_{n k}, c_{n k} ; 1-e^{-2 \alpha r}\right)}, \\
& a_{n k}= \eta+\beta+\sqrt{\frac{A}{\alpha}\left(E_{n k}+M\right)-\frac{E_{n k}^{2}-M^{2}}{4 \alpha^{2}}}, \\
& b_{n k}= \eta+\beta-\sqrt{\frac{A}{\alpha}\left(E_{n k}+M\right)-\frac{E_{n k}^{2}-M^{2}}{4 \alpha^{2}}} \\
& c_{n k}=2 \eta .
\end{aligned}
$$

According to (26), we obtain the following form of energy eigenvalue equation for scattering states:

$$
\eta+\beta-\sqrt{\frac{A}{\alpha}\left(E_{n k}+M\right)-\frac{E_{n k}^{2}-M^{2}}{4 \alpha^{2}}}=-n .
$$

Now, by finding the asymptotic form of (32) for large $r$, we try to obtain the scattering phase shifts. For this purpose, we use the following property of the hypergeometric function:

$$
\begin{aligned}
{ }_{2} F_{1}(a, b, c ; x) & \\
= & \frac{\Gamma(c) \Gamma(c-a-b)}{\Gamma(c-a) \Gamma(c-b)} \\
& \times{ }_{2} F_{1}(a, b ; a+b-c+1 ; 1-x) \\
& +(1-x)^{c-a-b} \frac{\Gamma(c) \Gamma(a+b-c)}{\Gamma(a) \Gamma(b)} \\
& \times{ }_{2} F_{1}(c-a, c-b ; c-a-b+1 ; 1-x), \\
& { }_{2} F_{1}(a, b, c, 0)=1 .
\end{aligned}
$$


Therefore, we obtain

$$
\begin{aligned}
{ }_{2} F_{1}\left(a, b, c ; 1-e^{-2 \alpha r}\right) \\
=\frac{\Gamma(c) \Gamma(c-a-b)}{\Gamma(c-a) \Gamma(c-b)} \\
\quad \times{ }_{2} F_{1}\left(a, b ; a+b-c+1 ; e^{-2 \alpha r}\right) \\
\quad+\left(e^{-2 \alpha r}\right)^{c-a-b} \frac{\Gamma(c) \Gamma(a+b-c)}{\Gamma(a) \Gamma(b)} \\
\quad \times{ }_{2} F_{1}\left(c-a, c-b ; c-a-b+1 ; e^{-2 \alpha r}\right) .
\end{aligned}
$$

By using this definition

$$
\beta=i k_{1}, \quad k_{1}=\sqrt{\frac{E_{n k}^{2}-M^{2}}{4 \alpha^{2}}}
$$

the upper component of wavefunction is as follows:

$$
\begin{aligned}
F_{n k}(r)= & N_{n k}\left(1-e^{-2 \alpha r}\right)^{\eta} e^{-2 i k_{1} \alpha r} \\
& \times{ }_{2} F_{1}\left(a_{n k}, b_{n k}, c_{n k} ; 1-e^{-2 \alpha r}\right) .
\end{aligned}
$$

Now, with (38) and (39) in the limit $r \rightarrow \infty$ we have

$$
\begin{aligned}
F_{n k}(r) \longrightarrow & N_{r \rightarrow \infty}\left(1-e^{-2 \alpha r}\right)^{\eta} e^{-2 i k_{1} \alpha r} \\
& \times\left\{\frac{\Gamma\left(c_{n k}\right) \Gamma\left(c_{n k}-a_{n k}-b_{n k}\right)}{\Gamma\left(c_{n k}-a_{n k}\right) \Gamma\left(c_{n k}-b_{n k}\right)}\right. \\
& \left.+\frac{\Gamma\left(c_{n k}\right) \Gamma\left(a_{n k}+b_{n k}-c_{n k}\right)}{\Gamma\left(a_{n k}\right) \Gamma\left(b_{n k}\right)} e^{4 i k_{1} \alpha r}\right\} .
\end{aligned}
$$

Then

$$
\begin{aligned}
F_{n k}(r \longrightarrow \infty) \longrightarrow & N_{n k} \Gamma\left(c_{n k}\right) \\
& \times\left|\frac{\Gamma\left(c_{n k}-a_{n k}-b_{n k}\right)}{\Gamma\left(c_{n k}-a_{n k}\right) \Gamma\left(c_{n k}-b_{n k}\right)}\right| \\
& \times\left(e^{-i\left(2 k_{1} \alpha r-\delta\right)}+e^{i\left(2 k_{1} \alpha r-\delta\right)}\right) \\
= & 2 N_{n k} \Gamma\left(c_{n k}\right)\left|\frac{\Gamma\left(c_{n k}-a_{n k}-b_{n k}\right)}{\Gamma\left(c_{n k}-a_{n k}\right) \Gamma\left(c_{n k}-b_{n k}\right)}\right| \\
& \times \cos \left(2 k_{1} \alpha r-\delta\right) \\
= & 2 N_{n k} \Gamma\left(c_{n k}\right)\left|\frac{\Gamma\left(c_{n k}-a_{n k}-b_{n k}\right)}{\Gamma\left(c_{n k}-a_{n k}\right) \Gamma\left(c_{n k}-b_{n k}\right)}\right| \\
& \times \sin \left(\delta-2 k_{1} \alpha r+\frac{\pi}{2}\right) .
\end{aligned}
$$

The general boundary condition of the scattering state wave function on the " $\left(k_{1} / 2 \pi\right)$ scale" is

$$
\varphi(r)=2 \sin \left(k_{1} r-\frac{\pi}{2} l+\delta_{l}\right) .
$$

Therefore, we have

$$
\begin{aligned}
F_{n k}(r & \longrightarrow \infty) \\
\longrightarrow & 2 N_{n k} \Gamma\left(c_{n k}\right) \\
& \times\left|\frac{\Gamma\left(c_{n k}-a_{n k}-b_{n k}\right)}{\Gamma\left(c_{n k}-a_{n k}\right) \Gamma\left(c_{n k}-b_{n k}\right)}\right| \\
& \times \sin \left(2 \alpha k_{1} r+\frac{\pi}{2}\right. \\
& \left.\quad+\arg \left(\frac{\Gamma\left(c_{n k}-a_{n k}-b_{n k}\right)}{\Gamma\left(c_{n k}-a_{n k}\right) \Gamma\left(c_{n k}-b_{n k}\right)}\right)\right) .
\end{aligned}
$$

Thus, we obtain the scattering phase shifts as follows:

$$
\begin{aligned}
\delta_{l, n k}= & \frac{\pi}{2}(l+1)+\arg \Gamma\left(c_{n k}-a_{n k}-b_{n k}\right) \\
& -\arg \Gamma\left(c_{n k}-a_{n k}\right)-\arg \Gamma\left(c_{n k}-b_{n k}\right) .
\end{aligned}
$$

Then we have the scattering phase shifts of the upper component of wavefunction as

$\delta_{l, n k}=\frac{\pi}{2}(l+1)+\arg \Gamma(2 \beta)$

$$
\begin{aligned}
& -\arg \Gamma\left(\eta+\beta-\sqrt{2 \eta^{2}+\beta^{2}+4 \eta \beta+\frac{A}{\alpha}\left(E_{n k}+M\right)}\right) \\
& -\arg \Gamma\left(\eta+\beta+\sqrt{2 \eta^{2}+\beta^{2}+4 \eta \beta+\frac{A}{\alpha}\left(E_{n k}+M\right)}\right) .
\end{aligned}
$$

For the lower component, with (34), we have

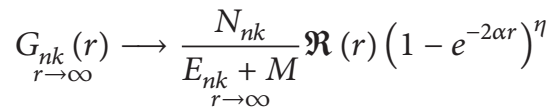

$$
\begin{aligned}
& \times e^{-2 i k_{1} \alpha r}\left\{\frac{\Gamma\left(c_{n k}\right) \Gamma\left(c_{n k}-a_{n k}-b_{n k}\right)}{\Gamma\left(c_{n k}-a_{n k}\right) \Gamma\left(c_{n k}-b_{n k}\right)}\right. \\
& +\frac{\Gamma\left(c_{n k}\right) \Gamma\left(a_{n k}+b_{n k}-c_{n k}\right)}{\Gamma\left(a_{n k}\right) \Gamma\left(b_{n k}\right)} \\
& \left.\times e^{4 i k_{1} \alpha r}\right\}
\end{aligned}
$$


TABLE 1: Energy eigenvalues of the Yukawa potential for different values of $n$ and $k$ (in $\hbar=c=1$ unit) for $M=0.5 f \mathrm{~m}^{-1}, A=0.1$, and $\alpha=0.01$.

\begin{tabular}{ccccccccc}
\hline$n$ & $l, k>0$ & $l, j=l-1 / 2$ & $v$ & $E_{k>0}$ & $l, k<0$ & $l, j=l+1 / 2$ & $v$ & $E_{k<0}$ \\
\hline 0 & 1,1 & $0 p_{1 / 2}$ & 2 & -0.4996006388 & $0,-1$ & $0 s_{1 / 2}$ & 1 & -0.4999001896 \\
0 & 2,2 & $0 d_{3 / 2}$ & 3 & -0.4991009891 & $1,-2$ & $0 p_{3 / 2}$ & 2 & -0.4996006388 \\
0 & 3,3 & $0 f_{5 / 2}$ & 4 & -0.4984006400 & $2,-3$ & $0 d_{5 / 2}$ & 3 & -0.4991009891 \\
0 & 4,4 & $0 g_{7 / 2}$ & 5 & -0.4974987420 & $3,-4$ & $0 f_{7 / 2}$ & 4 & -0.4984006400 \\
1 & 1,1 & $1 p_{1 / 2}$ & 2 & -0.4991009891 & $0,-1$ & $1 s_{1 / 2}$ & 1 & -0.4996006388 \\
1 & 2,2 & $1 d_{3 / 2}$ & 3 & -0.4984006400 & $1,-2$ & $1 p_{3 / 2}$ & 2 & -0.4991009891 \\
1 & 3,3 & $1 f_{5 / 2}$ & 4 & -0.4974987420 & $2,-3$ & $1 d_{5 / 2}$ & 3 & -0.4984006400 \\
1 & 4,4 & $1 g_{7 / 2}$ & 5 & -0.4963942080 & $3,-4$ & $1 f_{7 / 2}$ & 4 & -0.4974987420 \\
1 & 1,1 & $2 p_{1 / 2}$ & 2 & -0.4984006400 & $0,-1$ & $2 s_{1 / 2}$ & 1 & -0.4991009891 \\
2 & 2,2 & $2 d_{3 / 2}$ & 3 & -0.4974987420 & $1,-2$ & $2 p_{3 / 2}$ & 2 & -0.4984006400 \\
2 & 3,3 & $2 f_{5 / 2}$ & 4 & -0.4963942080 & $2,-3$ & $2 d_{5 / 2}$ & 3 & -0.4974987420 \\
2 & 4,4 & $2 g_{7 / 2}$ & 5 & -0.4950856720 & $3,-4$ & $2 f_{7 / 2}$ & 4 & -0.4963942080 \\
\hline
\end{tabular}

$$
\begin{aligned}
& \lim _{\substack{R \rightarrow \infty \\
r}} \\
& =\lim _{r \rightarrow \infty}\left(\frac{k}{r}-2 \alpha \beta+2 \alpha \eta e^{-2 \alpha r}\right. \\
& \\
& \quad \times\left(1-e^{-2 \alpha r}\right)^{-1}+\left(\frac{a_{n k} b_{n k}}{c_{n k}}\right) \\
& \\
& \left.\quad \times \frac{{ }_{2} F_{1}\left(a_{n k}+1, b_{n k}+1, c_{n k}+1 ; 1-e^{-2 \alpha r}\right)}{{ }_{2} F_{1}\left(a_{n k}, b_{n k}, c_{n k} ; 1-e^{-2 \alpha r}\right)}\right) \\
& =-2 \alpha \beta+\frac{a_{n k} b_{n k}}{c_{n k}} .
\end{aligned}
$$

Then we have

$$
\begin{aligned}
G_{n k}(r \longrightarrow \infty) \longrightarrow & H_{n k} \Gamma\left(c_{n k}\right) \\
& \times\left|\frac{\Gamma\left(c_{n k}-a_{n k}-b_{n k}\right)}{\Gamma\left(c_{n k}-a_{n k}\right) \Gamma\left(c_{n k}-b_{n k}\right)}\right| \\
& \times\left(e^{-i\left(2 k_{1} \alpha r-\delta\right)}+e^{i\left(2 k_{1} \alpha r-\delta\right)}\right),
\end{aligned}
$$

where

$$
H_{n k}=\frac{N_{n k}}{E_{n k}+M}\left(-2 \alpha \beta+\frac{a_{n k} b_{n k}}{c_{n k}}\right)
$$

Thus with (43), (44), (45), and (49), we have the following:

$$
\begin{aligned}
G_{n k}(r \longrightarrow \infty) & \\
\longrightarrow & 2 H_{n k} \Gamma\left(c_{n k}\right) \\
& \times\left|\frac{\Gamma\left(c_{n k}-a_{n k}-b_{n k}\right)}{\Gamma\left(c_{n k}-a_{n k}\right) \Gamma\left(c_{n k}-b_{n k}\right)}\right|
\end{aligned}
$$

$$
\begin{aligned}
\times \sin \left(2 \alpha k_{1} r+\frac{\pi}{2}\right. \\
\left.\quad+\arg \left(\frac{\Gamma\left(c_{n k}-a_{n k}-b_{n k}\right)}{\Gamma\left(c_{n k}-a_{n k}\right) \Gamma\left(c_{n k}-b_{n k}\right)}\right)\right) .
\end{aligned}
$$

And finally we have

$$
\begin{aligned}
\delta_{l, n k}= & \frac{\pi}{2}(l+1)+\arg \Gamma\left(c_{n k}-a_{n k}-b_{n k}\right) \\
& -\arg \Gamma\left(c_{n k}-a_{n k}\right)-\arg \Gamma\left(c_{n k}-b_{n k}\right) .
\end{aligned}
$$

Therefore, the scattering phase shifts of two components are equal but in a constant coefficient.

\section{Conclusions}

We have studied the Dirac equation with the Yukawa potential and have obtained bound and scattering states of this problem. The energy eigenvalues, eigenstates, and scattering phase shifts have been presented. The numerical results of the energy eigenvalues of this work have been compared with the ones obtained in the literature in Table 1.

\section{Conflict of Interests}

The authors declare that there is no conflict of interests regarding the publication of this paper.

\section{Acknowledgment}

The authors thank the anonymous referees for the valuable comments and suggestions. 


\section{References}

[1] J. Y. Guo and X. Z. Fang, "Scattering of a Klein-Gordon particle by a Hulthén potential," Canadian Journal of Physics, vol. 87, no. 9, pp. 1021-1024, 2009.

[2] W. C. Qiang and S. H. Dong, "Analytical approximations to the solutions of the Manning-Rosen potential with centrifugal term," Physics Letters A, vol. 368, no. 1-2, pp. 13-17, 2007.

[3] G. F. Wei and S. H. Dong, "Spin symmetry in the relativistic symmetrical well potential including a proper approximation to the spin-orbit coupling term," Physica Scripta, vol. 81, no. 3, Article ID 035009, 2010.

[4] G. F. Wei and S. H. Dong, "Algebraic approach to pseudospin symmetry for the Dirac equation with scalar and vector modified Pöschl-Teller potentials," Europhysics Letters, vol. 87, no. 4, Article ID 40004, 2009.

[5] G. F. Wei and S. H. Dong, "The spin symmetry for deformed generalized Pöschl-Teller potential," Physics Letters A, vol. 373, no. 29, pp. 2428-2431, 2009.

[6] G. F. Wei and S. H. Dong, "Approximately analytical solutions of the Manning-Rosen potential with the spin-orbit coupling term and spin symmetry," Physics Letters A, vol. 373, no. 1, pp. 49-53, 2008.

[7] G. F. Wei and S. H. Dong, "A novel algebraic approach to spin symmetry for Dirac equation with scalar and vector second Pöschl-Teller potentials," The European Physical Journal A, vol. 43, no. 2, pp. 185-190, 2010.

[8] G. F. Wei and S. H. Dong, "Pseudospin symmetry in the relativistic Manning-Rosen potential including a Pekeris-type approximation to the pseudo-centrifugal term," Physics Letters $B$, vol. 686, no. 4-5, pp. 288-292, 2010.

[9] G. F. Wei and S. H. Dong, "Pseudospin symmetry for modified Rosen-Morse potential including a Pekeris-type approximation to the pseudo-centrifugal term," The European Physical Journal A, vol. 46, no. 2, pp. 207-212, 2010.

[10] V. M. Villalba and L. A. Gonzalez-Arraga, "Tunneling and transmission resonances of a Dirac particle by a double barrier," Physica Scripta, vol. 81, no. 2, Article ID 025010, 2010.

[11] G. F. Wei, W. C. Qiang, and W. L. Chen, "Approximate analytical solution of continuous states for the l-wave Schrödinger equation with a diatomic molecule potential," Central European Journal of Physics, vol. 8, no. 4, pp. 574-579, 2010.

[12] G. F. Wei and W. L. Chen, "Continuum states of modified Morse potential," Chinese Physics B, vol. 19, no. 9, Article ID 090308, 2010.

[13] C. Rojas and V. M. Villalba, "Scattering of a Klein-Gordon particle by a Woods-Saxon potential," Physical Review A, vol. 71, no. 5, Article ID 052101, p. 4, 2005.

[14] C. Y. Chen, F. L. Lu, and D. S. Sun, "Exact solutions of scattering states for the s-wave Schrödinger equation with the ManningRosen potential," Physica Scripta, vol. 76, no. 5, pp. 428-430, 2007.

[15] N. Dombey and P. Kennedy, "Low momentum scattering in the Dirac equation," Journal of Physics A, vol. 35, no. 31, pp. 66456657, 2002.

[16] C. Y. Chen, D. S. Sun, and F. L. Lu, "Scattering states of the Klein-Gordon equation with Coulomb-like scalar plus vector potentials in arbitrary dimension," Physics Letters A, vol. 330, no. 6, pp. 424-428, 2004.

[17] W. Greiner, Relativistic Quantum Mechanics, Wave Equations, Springer, Berlin, Germany, 3rd edition, 2000.
[18] L. H. Zhang, X. P. Li, and C. S. Jia, "Analytical approximation to the solution of the Dirac equation with the Eckart potential including the spin-orbit coupling term," Physics Letters A, vol. 372, no. 13, pp. 2201-2207, 2008.

[19] C. S. Jia, P. Guo, and X. L. Peng, "Exact solution of the DiracEckart problem with spin and pseudospin symmetry," Journal of Physics A, vol. 39, no. 24, pp. 7737-7744, 2006.

[20] S. M. Ikhdair and R. Sever, "Exact solution of the KleinGordon equation for the PT-symmetric generalized WoodsSaxon potential by the Nikiforov-Uvarov method," Annalen der Physik, vol. 16, no. 3, pp. 218-232, 2007.

[21] H. Yukawa, "The prediction and discovery of pions and muons," Proceedings of the Physico-Mathematical Society of Japan, vol. 17, no. 3, p. 48, 1935.

[22] A. D. Alhaidari, H. Bahlouli, and M. S. Abdelmonem, “Taming the Yukawa potential singularity: improved evaluation of bound states and resonance energies," Journal of Physics A, vol. 41, no. 3, Article ID 032001, p. 9, 2008.

[23] R. Sever and C. Tezcan, "Hypervirial solution for the generalized exponential cosine-screened Coulomb potential," Physical Review A, vol. 41, no. 9, pp. 5250-5208, 1990.

[24] R. L. Green and C. Aldrich, "Variational wave functions for a screened Coulomb potential," Physical Review A, vol. 14, no. 6, pp. 2363-2366, 1976.

[25] M. Hamzavi, M. Movahedi, K. E. Thylwe, and A. A. Rajabi, "Approximate analytical solution of the Yukawa potential with arbitrary angular momenta," Chinese Physics Letters, vol. 29, no. 8, Article ID 080302, 2012.

[26] M. Abramowitz and I. A. Stegun, Eds., Handbook of Mathematical Functions with Formulas, Graphs, and Mathematical Tables, U.S. Department of Commerce, National Bureau of Standards, New York, NY, USA, 1965. 

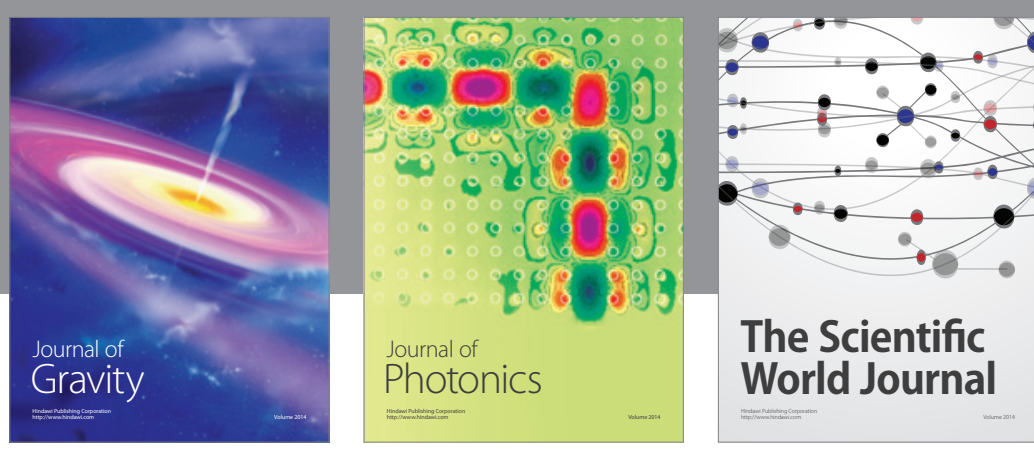

The Scientific World Journal
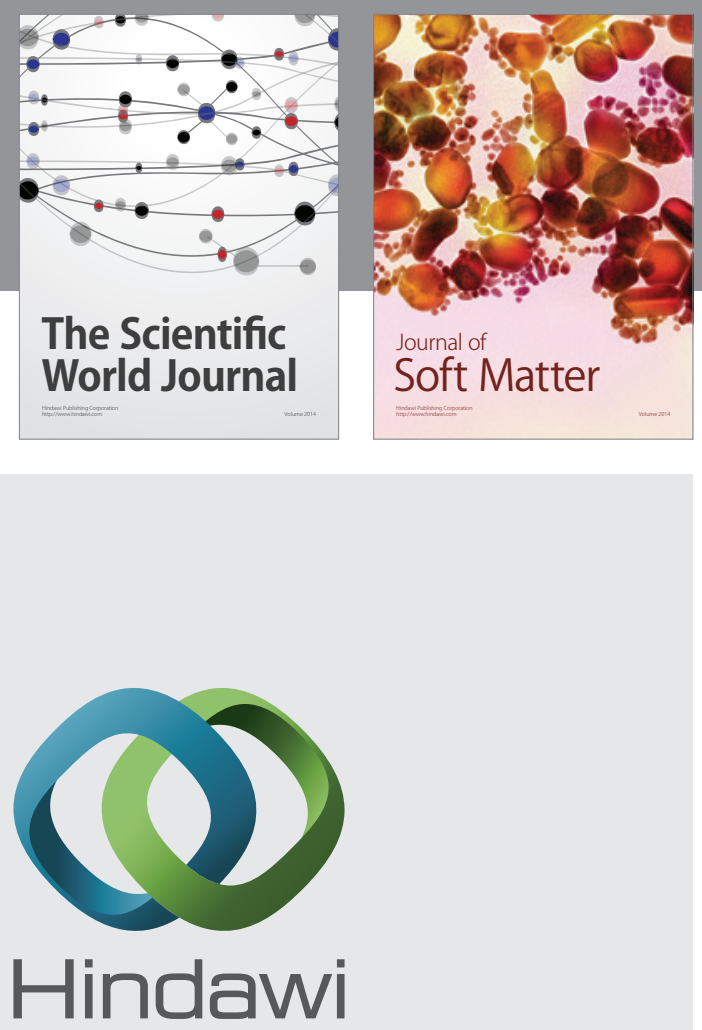

Submit your manuscripts at

http://www.hindawi.com

nternational Journal of

Statistical Mechanics
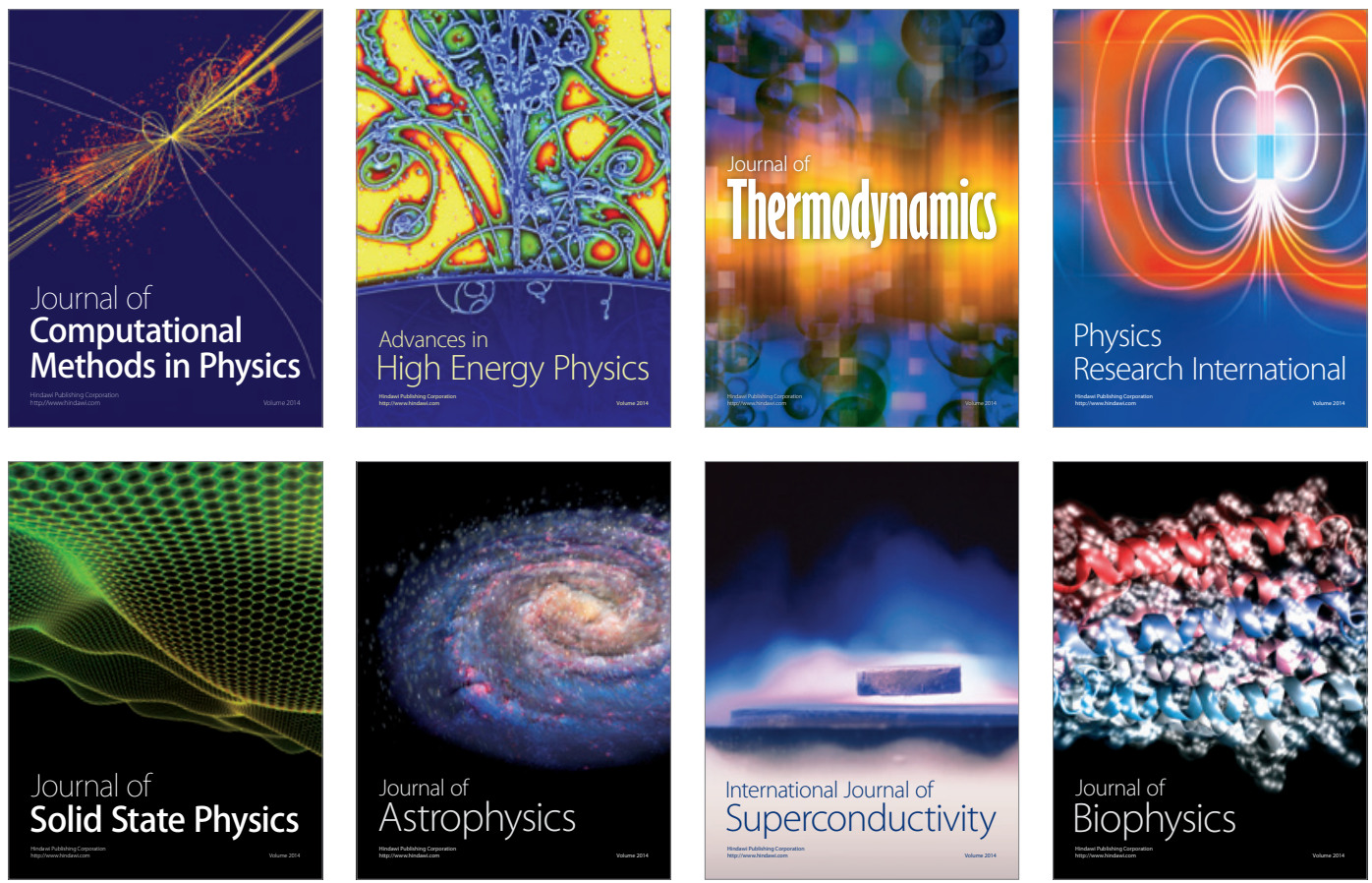
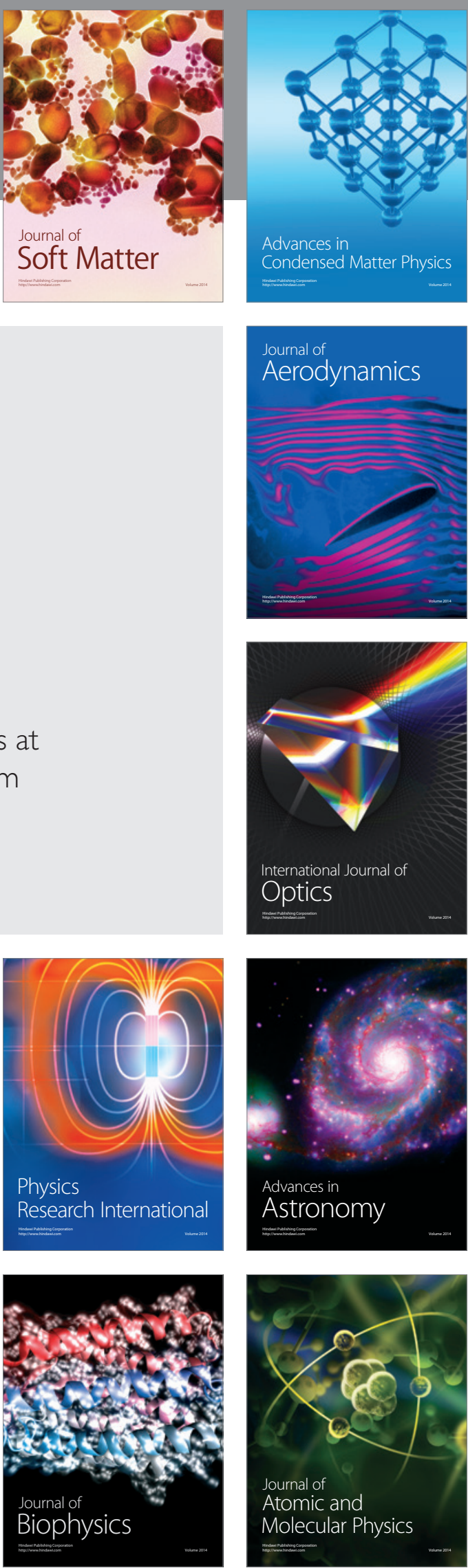